\title{
Accurate Solutions of Extremely Large Integral-Equation Problems in Computational Electromagnetics
}

\author{
This paper reviews accurate solutions for extremely large integral-equation \\ problems in computational electromagnetics.
}

By Özgür ErgüL, Member IEEE, And Levent Gürel, Fellow IEEE

\begin{abstract}
Accurate simulations of real-life electromagnetics problems with integral equations require the solution of dense matrix equations involving millions of unknowns. Solutions of these extremely large problems cannot be achieved easily, even when using the most powerful computers with state-ofthe-art technology. However, with the multilevel fast multipole algorithm (MLFMA) and parallel MLFMA, we have been able to obtain full-wave solutions of scattering problems discretized with hundreds of millions of unknowns. Some of the complicated real-life problems (such as scattering from a realistic aircraft) involve geometries that are larger than 1000 wavelengths. Accurate solutions of such problems can be used as benchmarking data for many purposes and even as reference data for high-frequency techniques. Solutions of extremely large canonical benchmark problems involving sphere and National Aeronautics and Space Administration (NASA) Almond geometries are presented, in addition to the solution of complicated objects, such as the Flamme. The parallel implementation is also extended to solve very large dielectric problems, such as dielectric lenses and photonic crystals.
\end{abstract}

Manuscript received July 24, 2011; revised December 2, 2011 and January 27, 2012; accepted January 30, 2012. Date of publication August 17, 2012; date of current version January 16,2013 . This work was supported by the Scientific and Technical Research Council of Turkey (TUBITAK) under Research Grants 110E268 and 111E203, by the Centre for Numerical Algorithms and Intelligent Software (EPSRC-EP/G036136/1), and by contracts from ASELSAN Inc. and Undersecretariat for Defense Industries (SSM). ö. Ergül is with the Department of Mathematics and Statistics, University of Strathclyde, Glasgow G11XH, U.K. (e-mail: ozgur.ergul@strath.ac.uk).

L. Gürel is with the Department of Electrical and Electronics Engineering and the Computational Electromagnetics Research Center (BiLCEM), Bilkent University, Bilkent, Ankara TR-06800, Turkey (e-mail: Igurel@bilkent.edu.tr).

Digital Object Identifier: 10.1109/JPROC.2012.2204429
KEYWORDS | Computational electromagnetics; iterative solutions; large-scale problems; multilevel fast multipole algorithm (MLFMA); parallelization; surface integral equations

\section{INTRODUCTION}

Real-life electromagnetics problems often involve large-scale objects discretized with millions of unknowns. These problems can be solved accurately and efficiently with the multilevel fast multipole algorithm (MLFMA) [1]-[3] Parallelization of MLFMA on parallel computers enables the solution of even larger problems [4]. Recently, various parallel implementations have been developed to increase the problem size from millions to more than one billion [5][17]. Thanks to advances in both computer hardware and parallelization techniques, solutions of electromagnetics problems involving realistic objects discretized with hundreds of millions of unknowns have become not only possible but also increasingly more common [17]-[19]. This paper presents an overview of a sophisticated simulation environment based on a parallel implementation of MLFMA that is capable of such large-scale solutions on moderately powerful computers. We present numerical examples involving both canonical and complicated targets that are larger than 1000 wavelengths. We also present the solution of large-scale dielectric problems, such as transmission through dielectric lenses and photonic crystals. Details of the parallel implementation can be found in [12] and [20].

Before presenting the details of large-scale simulations, we need to answer the following question: Is it required to solve large-scale electromagnetics problems using a full-wave 

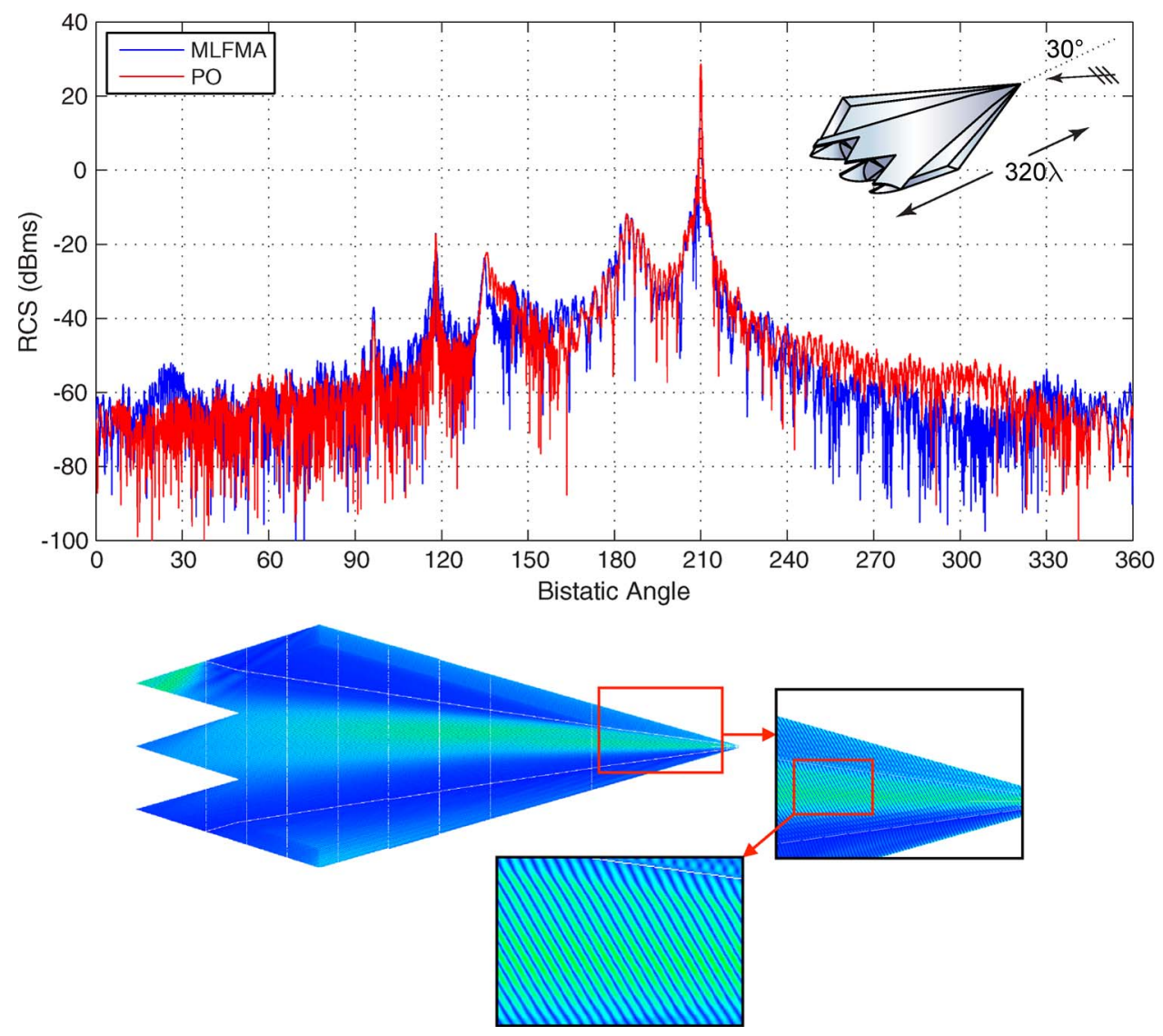

Fig. 1. Solutions of a scattering problem involving the Flamme at $160 \mathrm{GHz}$. Bistatic RCS (dBms) is plotted as a function of the observation angle from $O^{\circ}$ to $360^{\circ}$, where $30^{\circ}$ and $210^{\circ}$ correspond to the backscattering and forward-scattering directions, respectively. Imaginary part of the electric current induced on the object (calculated with MLFMA) is also depicted.

solver, considering that much more efficient high-frequency techniques are available in the literature? The answer depends on the level of desired accuracy. For example, Fig. 1 presents the solution of a scattering problem involving a stealth airborne target called the Flamme [21]. The target is located in free space and illuminated by a plane wave propagating at $30^{\circ}$ angle from the nose with the electric field polarized horizontally. The scaled size of the target is $0.6 \mathrm{~m}$, corresponding to approximately $320 \lambda$ at $160 \mathrm{GHz}$, where $\lambda$ is the wavelength. Fig. 1 shows the bistatic radar-cross-section (RCS) values of the Flamme in decibel meter squared (dBms) as a function of the bistatic angle. In the plot, $30^{\circ}$ and $210^{\circ}$ correspond to the backscattering and forward-scattering directions, respectively. RCS values are obtained with parallel MLFMA (with 1\% maximum error) in hours and with physical optics (PO) in seconds. From one point of view, $\mathrm{PO}$ is quite successful by providing reasonably accurate RCS values, especially in and around the forward-scattering direction. On the other hand, there are large discrepancies (as large as 10-20 dB) between the results in other directions. Furthermore, the oscillations with respect to the observation angle of the RCS values predicted by MLFMA and PO do not agree well with each other. This might be expected since PO is an approximate technique and it is based on the high-frequency behavior of electromagnetic waves. Unlike PO, the MLFMA solver takes all multiplescattering mechanisms into account. As a result, the backscattering RCS, which is a fundamentally important quantity, may be in error by as much as $14.75 \mathrm{~dB}$ with $\mathrm{PO}$, and this may not be acceptable for many applications. Fig. 1 also depicts the imaginary part of the electric current induced on the surface of the Flamme calculated with MLFMA. It can be observed that the current behaves as predicted by PO, e.g., there are lit and shadow regions and oscillations with a periodicity of the wavelength. Nevertheless, a detailed inspection reveals that the current and electromagnetic fields are actually more complicated, i.e., there are multiple reflections, diffractions, resonances, etc. All these phenomena can accurately be modeled only by using a full-wave solver, such as MLFMA.

It should be emphasized that full-wave solvers, including fast solvers (such as MLFMA), cannot compete (in terms of time) against the approximation techniques (such as PO) with today's computing technology. Approximation 
techniques are still thousands of times faster and most of them can be implemented on a desktop computer, without any need to employ high-performance computing. Nevertheless, full wave solvers are now capable of solving very large real-life problems with unprecedented levels of accuracy and details. In fact, results of full-wave simulations, including those presented in this paper, can be used as reference data to test the accuracy of various highfrequency techniques.

\section{SIMULATION ENVIRONMENT}

The simulation environment that is considered in this paper for full-wave solutions of large-scale electromagnetics problems in the frequency domain involves the following basic components:

- $\quad$ surface integral equations for efficient formulations;

- triangulations of surface geometries and low-order discretizations of unknown functions for flexible modeling and efficient numerical solutions;

- Krylov-subspace algorithms for fast iterative solutions;

- a robust implementation of MLFMA for fast matrix-vector multiplications, without sacrificing the accuracy;

- parallelization to solve extremely large electromagnetics problems.

This section provides an overview of these components, also considering the recent developments in the literature.

\section{A. Surface Integral Equations}

Electromagnetics problems involving metallic and dielectric objects can be formulated both rigorously and efficiently with surface integral equations [22]. For metallic objects, the electric-field integral equation (EFIE), the magnetic-field integral equation (MFIE), and the combined-field integral equation (CFIE) [23] are the most popular formulations. EFIE is applicable to open and closed surfaces, but it usually leads to ill-conditioned matrix equations and suffers from the well-known lowfrequency breakdown problem. Recent studies mainly focused on improving the conditioning of EFIE by using advanced discretizations based on decompositions [24], [25] and dual functions [26]. For closed objects, CFIE is preferred since it is free of the internal resonances [23] and less affected by the low-frequency breakdown problem. However, this formulation is contaminated with the inaccuracies due to the low-order discretizations of the identity operator [27], [28]. If the MFIE contribution is large in CFIE, compared to the EFIE contribution, accuracy of CFIE may be significantly hampered since MFIE contains an identity operator. Accuracy of CFIE can be improved by using the linear-linear functions [29].

Recently, novel integral-equation formulations for dielectric objects have been developed. Among them, the combined tangential formulation (CTF) [30], which is a rescaled version of the Poggio-Miller-Chang-Harrington-
$\mathrm{Wu}$-Tsai (PMCHWT) formulation [22], provides very accurate solutions with low-order discretizations. For better conditioned matrix equations, however, the electric and magnetic current combined-field integral equation (JMCFIE) [31]-[35] is preferred. This is a mixed formulation based on a linear combination of CTF and the combined normal formulation (CNF) [30]. Similar to CFIE for conducting objects, a Galerkin discretization of JMCFIE results in well-tested identity operators, which lead to very efficient iterative solutions [33], but may reduce the accuracy of the results. Therefore, the combination parameter in JMCFIE is extremely important for the tradeoff between the accuracy and the efficiency [32], [36].

\section{B. Discretization}

Probably, the most popular functions for low-order discretizations of the surface integral equations in computational electromagnetics are the Rao-WiltonGlisson (RWG) functions [37]. Despite their simplicity, the RWG functions provide the necessary flexibility to model the current distributions on arbitrarily complicated surfaces. They are also shown to possess the ability to properly model the charge distributions [38]. In order to use the RWG functions, surfaces are discretized with triangles that are small in terms of the wavelength. Dense matrix equations are obtained by expanding the currents and by testing the boundary conditions using a set of RWG functions. Electromagnetic interactions between the discretization elements (i.e., basis and testing functions) can be calculated via numerical integrations on triangular domains. Using the RWG functions, numerical integrations can be performed very accurately and efficiently [39]. The RWG functions are also very suitable for the acceleration methods based on the factorization and diagonalization, such as MLFMA. Unsurprisingly, some of the largest integral-equation problems solved in the literature are discretized with the RWG functions [5]-[17].

\section{Iterative Algorithms}

Many Krylov-subspace algorithms are available in the literature to perform iterative solutions of dense matrix equations derived from integral equations. Among them, the generalized minimal residual (GMRES) algorithm [40] has become popular due to its stability and efficiency for ill-conditioned matrix equations. Flexible variants of this algorithm have also been used to construct inner-outer schemes [41]. For well-conditioned matrix equations, however, alternative methods, such as the biconjugategradient-stabilized (BiCGStab) algorithm [42], can be more efficient than GMRES by providing fast convergence without increasing the memory requirement. BiCGStab is particularly useful for the matrix equations derived from CFIE and JMCFIE [5], [33]. Solutions of these formulations can be further accelerated with simple block-diagonal preconditioners [1], [33] that are suitable for low-complexity algorithms, such as MLFMA. 


\section{Multilevel Fast Multipole Algorithm}

Matrix-vector multiplications required for iterative solutions can be performed efficiently and accurately with MLFMA [1]-[3]. Using the factorization and diagonalization of electromagnetic interactions [43], MLFMA performs the multiplications of $N \times N$ matrices with arbitrary vectors in $\mathcal{O}(N \log N)$ time and using $\mathcal{O}(N \log N)$ memory. Each matrix-vector multiplication is formulated as a sequence of aggregation, translation, and disaggregation stages, which are realized on a multilevel tree structure that is constructed by the recursive clustering of the object. MLFMA implementations have various controllable error sources, in addition to the discretization, numerical integration, and iterative-residual errors. Specifically, two important accuracy parameters are the number of harmonics for the factorization/diagonalization and the size of stencils to interpolate/anterpolate [44] electromagnetic fields. The number of harmonics can be determined by the excess bandwidth formulas [45], [46], whereas the number of interpolation points can be determined heuristically [47]. The latter is relatively easy, considering that the same stencil can be applied at all levels of the tree structure. Global (instead of local) interpolation [48] is also an option to suppress the error if $\mathcal{O}\left(N \log ^{2} N\right)$ complexity is acceptable.

The conventional MLFMA has a low-frequency breakdown that inhibits its applicability to nonuniform and/or dense discretizations [49]. A straightforward solution to this problem is to discard the diagonalization (that is responsible for the breakdown) and employ multipole expansions directly for the subwavelength interactions [49]-[52].

\section{E. Parallelization}

Solutions of large-scale problems require the parallelization of MLFMA on parallel computers [4]-[20]. The idea is to distribute the computational tasks into several processes so that the solution can be performed quickly. In addition, parallelization enables the solution of larger problems by increasing the total memory available for solutions. Unfortunately, the parallelization of MLFMA is a formidably challenging mission due to complicated structure of this algorithm. Different parallelization schemes have appeared in the literature, especially for distributed-memory architectures, to increase the parallelization efficiency [4]-[17]. A successful technique is the hierarchical partitioning strategy [6], [10], [12], [20], which facilitates highly efficient parallelizations on hundreds of processors. The hierarchical strategy is based on optimizing the (subdomain and field) partitioning at each level of MLFMA such that the overall tree structure is distributed among processors in the best possible manner. Using the hierarchical strategy, the load balancing is improved and communications are accelerated. In this work, we use the hierarchical strategy for the solution of extremely large electromagnetics problems discretized

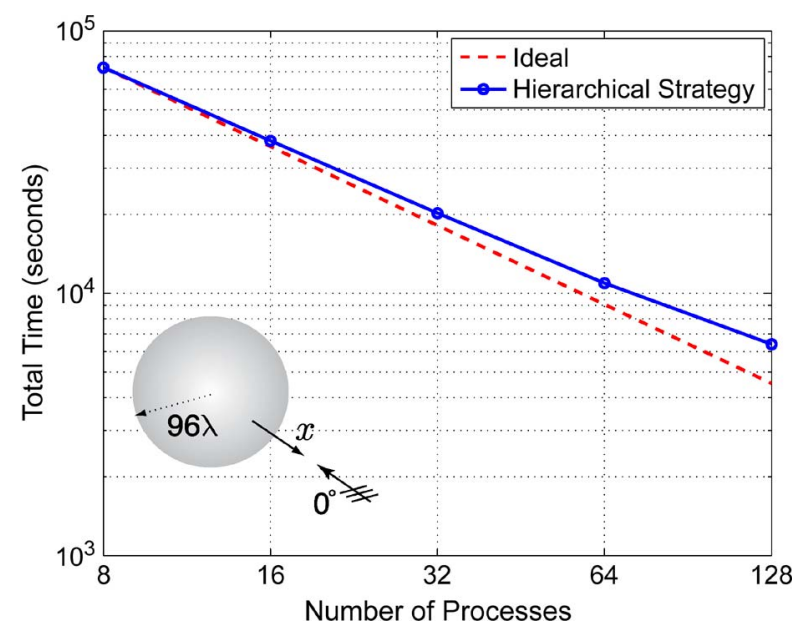

Fig. 2. Solutions of a scattering problem involving a conducting sphere of radius of $96 \lambda$ discretized with 33791232 unknowns. The total computing time is plotted as a function of the number of processes from 8 to 128.

with hundreds of millions of unknowns on moderate-size computers.

In order to demonstrate the improved parallelization efficiency with the hierarchical strategy, Fig. 2 presents solutions of a scattering problem involving a conducting sphere of radius $96 \lambda$ discretized with 33791232 unknowns. The problem is solved with a maximum of $1 \%$ error in the scattered fields. The solution is parallelized into $8,16,32,64$, and 128 processes on a cluster of Intel Xeon Nehalem-EX L7555 processors with 1.87-GHz clock rate. Fig. 2 depicts the total computing time, including problem input/output, setup, and 39 BiCGStab iterations (for 0.001 residual error). The ideal case assuming 100\% parallelization efficiency is also shown for comparisons. We observe that the computing time is significantly reduced from $72591 \mathrm{~s}$ to only $6400 \mathrm{~s}$ when the number of processes is increased from 8 to 128 .

\section{NUMERICAL EXAMPLES}

In this section, we present examples to the solution of large-scale electromagnetics problems with parallel MLFMA. All objects are located in free space, and the ensuing electromagnetics problems are formulated with CFIE or JMCFIE with a combination parameter of 0.5, i.e., $\mathrm{CFIE}=0.5 \times \mathrm{EFIE}+0.5 \times \mathrm{MFIE}$ and $\mathrm{JMCFIE}=0.5 \times$ $\mathrm{CTF}+0.5 \times \mathrm{CNF}$. All solutions are performed with a target of $1 \%$ maximum error in the scattered fields. In order to reach this target, all components of the error sources are kept well below 1\%. For example, numerical integrations of matrix elements (near-field and far-field interactions) are calculated with maximum 1\% error, and, for each problem, iterations are carried out until the residual error is reduced to below 0.001 or 0.005 . 
Similarly, other sources of error due to MLFMA and CFIE are also monitored and kept under $1 \%$.

\section{A. Benchmark Problems}

Fig. 3 presents the solution of a scattering problem involving a conducting sphere of radius $340 \lambda$ discretized with 540659712 unknowns. For the solution, MLFMA is parallelized into 64 processes on a cluster of Intel Xeon Nehalem-EX L7555 processors with $1.87-\mathrm{GHz}$ clock rate. The total computing time (including input/output, setup, and 65 BiCGStab iterations for 0.001 residual error) is $60.5 \mathrm{~h}$. The normalized bistatic RCS (i.e., $\mathrm{RCS} / \pi a^{2}$, where $a$ is the radius in meters) is plotted as a function of the observation angle from $0^{\circ}$ to $180^{\circ}$, where $0^{\circ}$ and $180^{\circ}$ correspond to the backscattering and forward-scattering directions, respectively. RCS values around the backscattering and forward-scattering directions are zoomed in. We
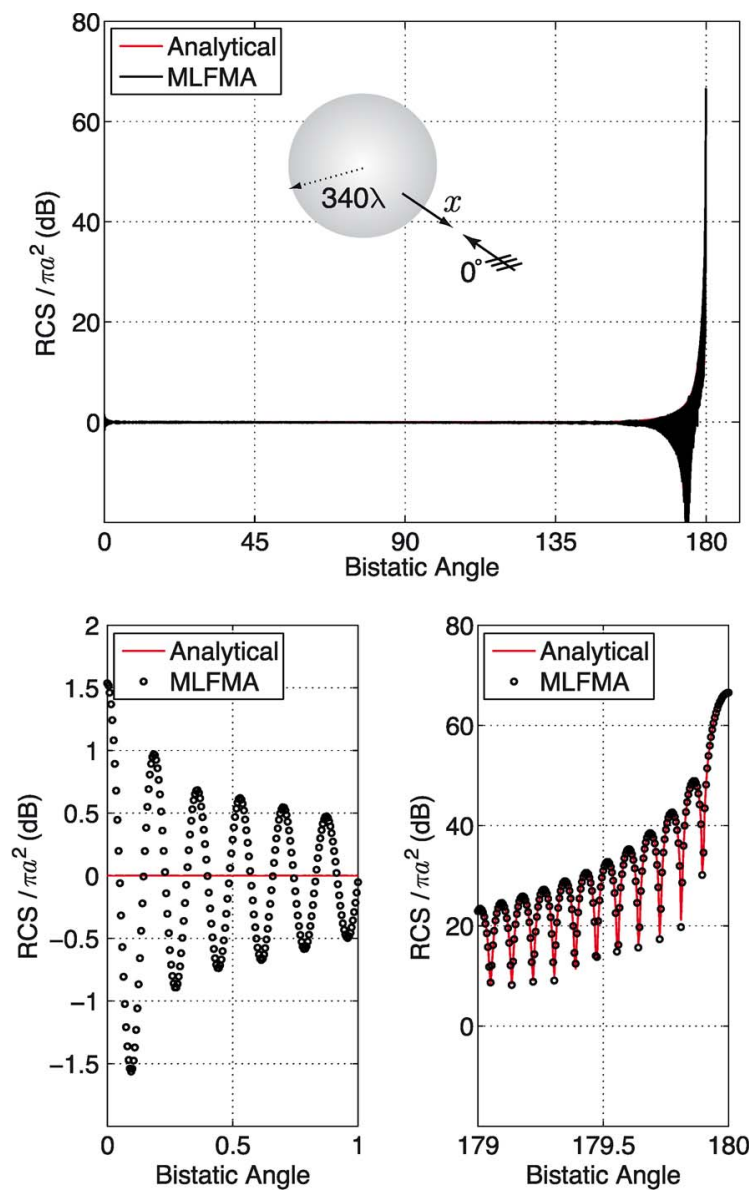

Fig. 3. Normalized bistatic RCS (RCS/ $\left.\pi \mathrm{a}^{2}\right)$ values of a sphere with a radius of $340 \lambda$ discretized with 540659712 unknowns from $O^{\circ}$ to $180^{\circ}$, where $O^{\circ}$ and $180^{\circ}$ correspond to the backscattering and forward-scattering directions, respectively. RCS values are zoomed in around the backscattering and forward-scattering directions in separate plots. Computational values provided by the parallel MLFMA implementation with $1 \%$ maximum error agree well with an analytical Mie-series solution.

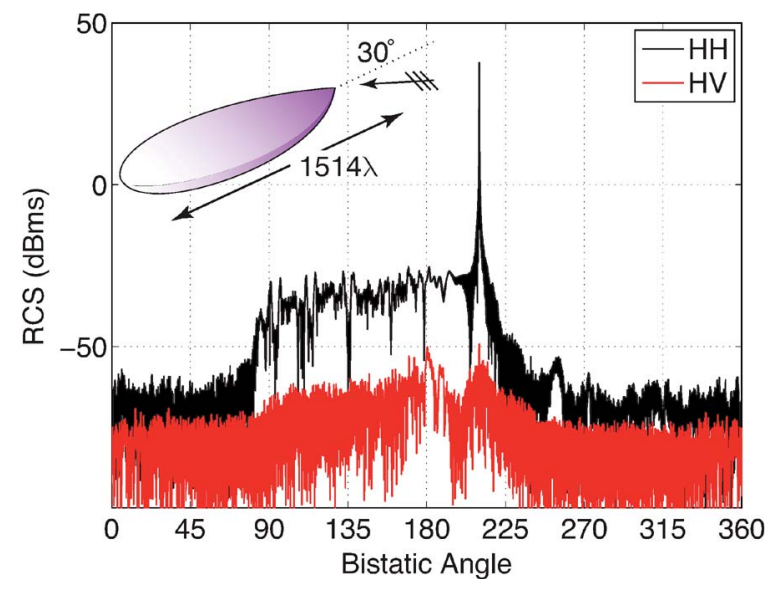

Fig. 4. Co-polar (HH) and cross-polar (HV) bistatic RCS values (dBms) of the NASA Almond at $1.8 \mathrm{THz}$. The target is illuminated by a plane wave propagating at $30^{\circ}$ angle from its nose with the electric field polarized horizontally. For numerical solutions, the target is discretized with 552310272 unknowns.

observe that the computational values agree very well with the analytical Mie-series solution. Considering all observation angles $\left(0^{\circ}\right.$ to $\left.180^{\circ}\right)$, the relative error (as calculated in [18]) is found to be $0.84 \%$. Assessing the accuracy of solutions is of the utmost importance. For this purpose, an interactive web-based benchmarking tool is prepared and made available at www.cem.bilkent.edu.tr/benchmark [54].

Fig. 4 presents the solution of a scattering problem involving another canonical object, i.e., the National Aeronautics and Space Administration (NASA) Almond [53], at $1.8 \mathrm{THz}$. The size of the target is approximately $1514 \lambda$ at this frequency. As depicted in the inset of the figure, the NASA Almond is illuminated by a plane wave propagating at $30^{\circ}$ angle from the nose with the electric field polarized horizontally. The problem is discretized with 552310272 unknowns and solved by using MLFMA on a cluster of Intel Xeon Nehalem-EX L7555 processors with $1.87-\mathrm{GHz}$ clock rate. The total computing time (including input/output, setup, and 70 BiCGStab iterations for 0.001 residual error) is $56.6 \mathrm{~h}$, when the solution is parallelized into 64 processes. Fig. 4 depicts the co-polar and cross-polar RCS values ( $\mathrm{dBms}$ ) with respect to the observation angle from $0^{\circ}$ to $360^{\circ}$, where $210^{\circ}$ corresponds to the forward-scattering direction. Typical properties of the RCS of the NASA Almond are observed: low backscattered RCS, no specular reflection, and relatively large values in the $90^{\circ}-210^{\circ}$ range.

\section{B. Flamme}

As an example of the application of MLFMA to complicated 3-D objects, Fig. 5 presents the solution of a scattering problem involving the stealth airborne target Flamme [21]. Similar to the NASA Almond, the target is illuminated by a plane wave propagating at $30^{\circ}$ angle from 


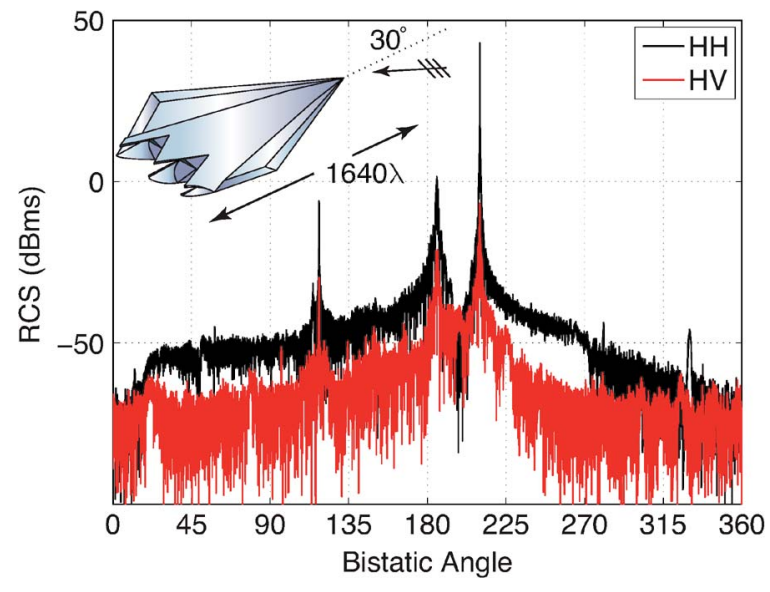

Fig. 5. Co-polar (HH) and cross-polar (HV) bistatic RCS values (dBms) of the Flamme at $820 \mathrm{GHz}$. The target is illuminated by a plane wave propagating at $30^{\circ}$ angle from its nose with the electric field polarized horizontally. For numerical solutions, the target is discretized with 538967040 unknowns.

the nose with the electric field polarized horizontally. At $820 \mathrm{GHz}$, the size of the target corresponds to approximately $1640 \lambda$ and it is discretized with 538967040 unknowns. For the solution, MLFMA is parallelized into 64 processes on a cluster of Intel Xeon Nehalem-EX L7555 processors with $1.87-\mathrm{GHz}$ clock rate. The total computing time (including input/output, setup, and 75 BiCGStab iterations for 0.001 residual error) is $58.2 \mathrm{~h}$. Fig. 5 depicts the co-polar and crosspolar RCS (dBms) values with respect to the observation angle from $0^{\circ}$ to $360^{\circ}$. The backscattered RCS (at $30^{\circ}$ ) is very low since the target exhibits stealth properties. On the other hand, there are specular reflections and the RCS of the Flamme makes peaks at several observation angles, in contrast with the RCS of the NASA Almond.

\section{Dielectric Problems}

The hierarchical parallelization of MLFMA can be extended to dielectric problems [35]. Fig. 6 presents the solution of an electromagnetics problem involving a dielectric hemisphere lens with a radius of $25 \mathrm{~mm}$. The lens has a relative permittivity of 4.8 and it is illuminated by a plane wave propagating toward its convex surface at $960 \mathrm{GHz}$. The problem is discretized with 39389184 unknowns and the solution is parallelized into 64 processes on a cluster of Intel Xeon Nehalem X5560 processors with $2.80-\mathrm{GHz}$ clock rate. The total computing time (including input/output, setup, and 68 BiCGStab iterations for 0.005 residual error) is $21.6 \mathrm{~h}$. Fig. 6 depicts the total electric field on the axis of rotation of the lens from $z=-40$ to $40 \mathrm{~mm}$. Focusing due to the lens is observed in the transmission region at around $z=-7 \mathrm{~mm}$, where the total electric field is maximum.

Finally, Fig. 7 presents the solution of a transmission problem involving a simple photonic crystal. Five dielectric

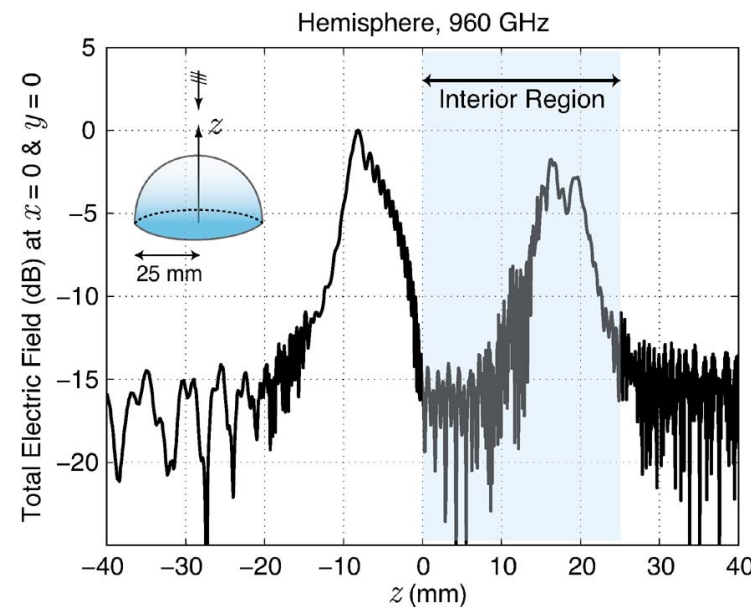

Fig. 6. Solution of an electromagnetics problem involving a dielectric hemisphere lens with a radius of $25 \mathrm{~mm}$ at $960 \mathrm{GHz}$. The lens has a relative permittivity of $\mathbf{4 . 8}$ and the problem is discretized with 39389184 unknowns. The total electric field on the axis of rotation of the lens is plotted from z $=-40$ to $40 \mathrm{~mm}$.

slabs of dimensions $2 \times 2 \times 0.41 \mathrm{~cm}^{3}$ are placed at $0.5-\mathrm{cm}$ intervals and illuminated by a plane wave at $960 \mathrm{GHz}$. The relative permittivity of the structure is 1.6 . The problem is discretized with 39628800 unknowns and the solution is parallelized into 64 processes on a cluster of Intel Xeon Nehalem X5560 processors with $2.80-\mathrm{GHz}$ clock rate. The total computing time (including input/output, setup, and 54 BiCGStab iterations for 0.005 residual error) is $10.1 \mathrm{~h}$. Fig. 7 depicts the electric field on the axis of symmetry from $z=-5$ to $5 \mathrm{~cm}$ to demonstrate the complicated electromagnetics response of the structure.

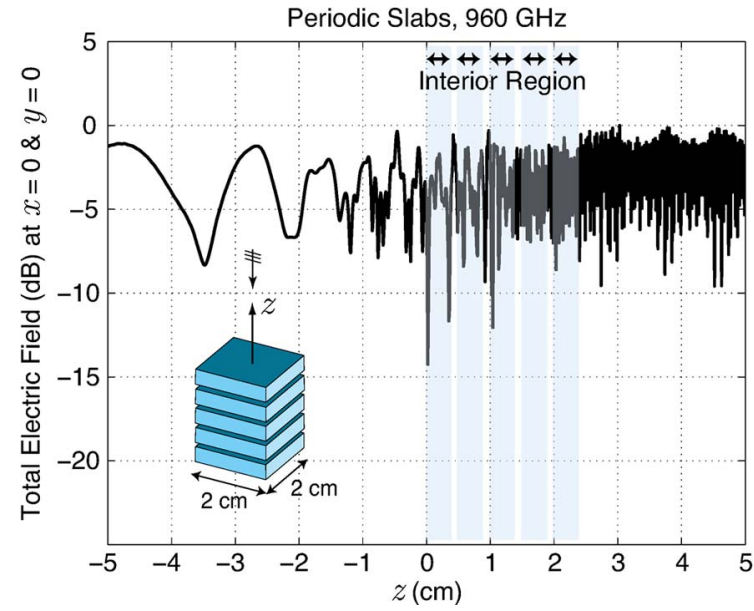

Fig. 7. Solution of an electromagnetics problem involving five $2 \times 2 \times$ $0.41-\mathrm{cm}^{3}$ dielectric slabs at $960 \mathrm{GHz}$. The structure has a relative permittivity of 1.6 and the problem is discretized with 39628800 unknowns. The total electric field on the axis of symmetry is plotted from $z=-5$ to $5 \mathrm{~cm}$. 


\section{CONCLUSION}

Fast and accurate solutions of extremely large integralequation problems are presented. By parallelizing MLFMA with the hierarchical partitioning strategy, it is possible to solve realistic electromagnetics problems involving 3-D complicated objects discretized with hundreds of millions of unknowns. Solutions that are obtained by a full-wave solver can be used for a variety of benchmarking purposes, including as reference data for approximate high-frequency techniques. Solutions of a set of extremely large problems are available at www.cem. bilkent.edu.tr/benchmark [54].

\section{Acknowledgment}

The authors would like to thank J. Wilcox of Intel Corporation for the expert technical support and the invaluable assistance he provided to facilitate the experiments on parallel computers. Computer time was provided in part by generous allocations from Intel Corporation.

\section{REFERENCES}

[1] J. Song, C.-C. Lu, and W. C. Chew, "Multilevel fast multipole algorithm for electromagnetic scattering by large complex objects," IEEE Trans. Antennas Propag., vol. 45, no. 10, pp. 1488-1493, Oct. 1997.

[2] X.-Q. Sheng, J.-M. Jin, J. Song, W. C. Chew, and C.-C. Lu, "Solution of combined-field integral equation using multilevel fast multipole algorithm for scattering by homogeneous bodies," IEEE Trans. Antennas Propag., vol. 46, no. 11, pp. 1718-1726, Nov. 1998.

[3] W. C. Chew, J.-M. Jin, E. Michielssen, and J. Song, Fast and Efficient Algorithms in Computational Electromagnetics. Boston, MA: Artech House, 2001.

[4] S. Velamparambil, W. C. Chew, and J. Song, "10 million unknowns: Is it that big?" IEEE Antennas Propag. Mag., vol. 45, no. 2, pp. 43-58, Apr. 2003.

[5] L. Gürel and Ö. Ergül, "Fast and accurate solutions of integral-equation formulations discretised with tens of millions of unknowns," Electron. Lett., vol. 43, no. 9, pp. 499-500, Apr. 2007.

[6] Ö. Ergül and L. Gürel, "Hierarchical parallelisation strategy for multilevel fast multipole algorithm in computational electromagnetics," Electron. Lett., vol. 44, no. 1, pp. 3-5, Jan. 2008.

[7] X.-M. Pan and X.-Q. Sheng, "A sophisticated parallel MLFMA for scattering by extremely large targets," IEEE Antennas Propag. Mag. vol. 50, no. 3, pp. 129-138, Jun. 2008.

[8] Ö. Ergül and L. Gürel, "Efficient parallelization of the multilevel fast multipole algorithm for the solution of large-scale scattering problems," IEEE Trans. Antennas Propag., vol. 56, no. 8, pp. 2335-2345, Aug. 2008.

[9] J. Fostier and F. Olyslager, "An asynchronous parallel MLFMA for scattering at multiple dielectric objects," IEEE Trans. Antennas Propag., vol. 56, no. 8, pp. 2346-2355, Aug. 2008.

[10] J. Fostier and F. Olyslager, "Provably scalable parallel multilevel fast multipole algorithm," Electron. Lett., vol. 44, no. 19, pp. 1111-1113, Sep. 2008.

[11] J. Fostier and F. Olyslager, "Full-wave electromagnetic scattering at extremely large 2-D objects," Electron. Lett., vol. 45, no. 5, pp. 245-246, Feb. 2009.

[12] Ö. Ergül and L. Gürel, "A hierarchical partitioning strategy for an efficient parallelization of the multilevel fast multipole algorithm," IEEE Trans. Antennas Propag., vol. 57, no. 6, pp. 1740-1750, Jun. 2009.

[13] J. M. Taboada, L. Landesa, F. Obelleiro, J. L. Rodriguez, J. M. Bertolo, M. G. Araujo, J. C. Mourino, and A. Gomez, "High scalability FMM-FFT electromagnetic solver for supercomputer systems," IEEE Antennas Propag. Mag., vol. 51, no. 6, pp. 21-28, Dec. 2009.

[14] M. G. Araujo, J. M. Taboada, F. Obelleiro, J. M. Bertolo, L. Landesa, J. Rivero, and J. L. Rodriguez, "Supercomputer aware approach for the solution of challenging electromagnetic problems," Progr. Electromagn. Res., vol. 101, pp. 241-256, 2010.

[15] J. M. Taboada, M. G. Araujo, J. M. Bertolo, L. Landesa, F. Obelleiro, and J. L. Rodriguez, "MLFMA-FFT parallel algorithm for the solution of large-scale problems in electromagnetics," Progr. Electromagn. Res., vol. 105, pp. 15-30, 2010.

[16] X.-M. Pan, W.-C. Pi, and X.-Q. Sheng, "On openmp parallelization of the multilevel fast multipole algorithm," Progr. Electromagn. Res. vol. 112, pp. 199-213, 2011.

[17] Ö. Ergül and L. Gürel, "Rigorous solutions of electromagnetics problems involving hundreds of millions of unknowns," IEEE Antennas Propag. Mag., vol. 53, no. 1, pp. 18-26, Feb. 2011.

[18] Ö. Ergül and L. Gürel, "Accuracy: The frequently overlooked parameter in the solution of extremely large problems," in Proc. Eur. Conf. Antennas Propag., 2011, pp. 3928-3931.

[19] Ö. Ergül and L. Gürel, "Benchmark solutions of large problems for evaluating accuracy and efficiency of electromagnetics solvers," in Proc. IEEE Antennas Propag. Soc. Int. Symp. 2011, pp. 179-182.

[20] L. Gürel and Ö. Ergül, "Hierarchical parallelization of the multilevel fast multipole algorithm (MLFMA)," Proc. IEEE, 2011.

[21] L. Gürel, H. Bağc1, J. C. Castelli, A. Cheraly, and F. Tardivel, "Validation through comparison: Measurement and calculation of the bistatic radar cross section (BRCS) of a stealth target," Radio Sci., vol. 38, no. 3, Jun. 2003.

[22] A. J. Poggio and E. K. Miller, "Integral equation solutions of three-dimensional scattering problems," in Computer Techniques for Electromagnetics, R. Mittra, Ed. Oxford, U.K.: Pergamon, 1973.

[23] J. R. Mautz and R. F. Harrington, "H-field, E-field, and combined field solutions for conducting bodies of revolution," AEÜ, vol. 32, no. 4, pp. 157-164, Apr. 1978.

[24] J.-F. Lee, R. Lee, and R. J. Burkholder, "Loop star basis functions and a robust preconditioner for EFIE scattering problems," IEEE Trans. Antennas Propag., vol. 51, no. 8, pp. 1855-1863, Aug. 2003.

[25] M. Taskinen and P. Ylä-Oijala, "Current and charge integral equation formulation," IEEE Trans. Antennas Propag., vol. 54, no. 1, pp. 58-67, Jan. 2006.

[26] F. P. Andriulli, K. Cools, H. Bagc1, F. Olyslager, A. Buffa, S. Christiansen, and
E. Michielssen, “A multiplicative Calderon preconditioner for the electric field integral equation," IEEE Trans. Antennas Propag., vol. 56, no. 8, pp. 2398-2412, Aug. 2008.

[27] Ö. Ergül and L. Gürel, "Discretization error due to the identity operator in surface integral equations," Comput. Phys. Commun., vol. 180, no. 10, pp. 1746-1752, Oct. 2009.

[28] L. Gürel and Ö. Ergül, “Contamination of the accuracy of the combined-field integral equation with the discretization error of the magnetic-field integral equation," IEEE Trans. Antennas Propag., vol. 57, no. 9 , pp. 2650-2657, Sep. 2009.

[29] Ö. Ergül and L. Gürel, "Linear-linear basis functions for MLFMA solutions of magnetic-field and combined-field integral equations," IEEE Trans. Antennas Propag., vol. 55, no. 4, pp. 1103-1110, Apr. 2007.

[30] P. Ylä-Oijala, M. Taskinen, and S. Järvenpää "Surface integral equation formulations for solving electromagnetic scattering problems with iterative methods," Radio Sci., vol. 40, Nov. 2005, DOI: 10.1029/2004RS003169, RS6002.

[31] P. Ylä-Oijala and M. Taskinen, “Application of combined field integral equation for electromagnetic scattering by dielectric and composite objects," IEEE Trans. Antennas Propag., vol. 53, no. 3, pp. 1168-1173, Mar. 2005.

[32] P. Ylä-Oijala, "Numerical analysis of combined field integral equation formulations for electromagnetic scattering by dielectric and composite objects," Progr. Electromagn. Res. C, vol. 3, pp. 19-43, 2008.

[33] Ö. Ergül and L. Gürel, "Comparison of integral-equation formulations for the fast and accurate solution of scattering problems involving dielectric objects with the multilevel fast multipole algorithm," IEEE Trans. Antennas Propag., vol. 57, no. 1, pp. 176-187, Jan. 2009.

[34] Ö. Ergül and L. Gürel, "Efficient solution of the electric and magnetic current combined-field integral equation with the multilevel fast multipole algorithm and block-diagonal preconditioning," Radio Sci., vol. 44, Nov. 2009, DOI: 10.1029/ 2009RS004143, RS6001.

[35] Ö. Ergül, "Solutions of large-scale electromagnetics problems involving dielectric objects with the parallel multilevel fast multipole algorithm," J. Opt. Soc. Amer. A. vol. 28, no. 11, pp. 2261-2268, Nov. 2011.

[36] Ö. Ergül, "Fast and accurate solutions of electromagnetics problems involving lossy dielectric objects with the multilevel fast multipole algorithm," Eng. Anal. Boundary Elements, vol. 36, no. 3, pp. 423-432, Mar. 2012.

[37] S. M. Rao, D. R. Wilton, and A. W. Glisson, "Electromagnetic scattering by surfaces of 
arbitrary shape," IEEE Trans. Antennas Propag., vol. AP-30, no. 3, pp. 409-418, May 1982.

[38] L. Gürel, K. Sertel, and I. K. Şendur, "On the choice of basis functions to model surface electric current densities in computational electromagnetics," Radio Sci., vol. 34, no. 6, pp. 1373-1387, Nov.-Dec. 1999.

[39] R. D. Graglia, "On the numerical integration of the linear shape functions times the 3-D Green's function or its gradient on a plane triangle," IEEE Trans. Antennas Propag., vol. 41, no. 10, pp. 1448-1455, Oct. 1993.

[40] Y. Saad and M. H. Schultz, "GMRES: A generalized minimal residual algorithm for solving nonsymmetric linear systems," SIAM J. Sci. Stat. Comput., vol. 7, no. 3, pp. 856-869, 1986.

[41] Ö. Ergül, T. Malas, and L. Gürel, "Solutions of large-scale electromagnetics problems using an iterative inner-outer scheme with ordinary and approximate multilevel fast multipole algorithms," Progr. Electromagn. Res., vol. 106, pp. 203-223, 2010.

[42] H. van der Vorst, "Bi-CGSTAB: A fast and smoothly converging variant of Bi-CG for the solution of nonsymmetric linear systems,"
SIAM J. Sci. Stat. Comput., vol. 13, no. 2, pp. 631-644, Mar. 1992.

[43] R. Coifman, V. Rokhlin, and S. Wandzura "The fast multipole method for the wave equation: A pedestrian prescription," IEEE Antennas Propag. Mag., vol. 35, no. 3, pp. 7-12, Jun. 1993.

[44] A. Brandt, "Multilevel computations of integral transforms and particle interactions with oscillatory kernels," Comput. Phys. Commun., vol. 65, pp. 24-38, Apr. 1991.

[45] S. Koc, J. M. Song, and W. C. Chew, "Error analysis for the numerical evaluation of the diagonal forms of the scalar spherical addition theorem," SIAM J. Numer. Anal., vol. 36, no. 3, pp. 906-921, 1999.

[46] S. Ohnuki and W. C. Chew, "Truncation error analysis of multipole expansions," SIAM J. Sci. Comput., vol. 25, no. 4, pp. 1293-1306, 2003.

[47] Ö. Ergül and L. Gürel, "Enhancing the accuracy of the interpolations and anterpolations in MLFMA," IEEE Antennas Wireless Propag. Lett., vol. 5, pp. 467-470, 2006.

[48] J. Sarvas, "Performing interpolation and anterpolation entirely by fast Fourier transform in the 3-D multilevel fas multipole algorithm," SIAM J. Numer. Anal. vol. 41, no. 6, pp. 2180-2196, Nov. 2003.

[49] J.-S. Zhao and W. C. Chew, "Three dimensional multilevel fast multipole algorithm from static to electrodynamic," Microw. Opt. Technol. Lett., vol. 26, no. 1 , pp. 43-48, Jul. 2000.

[50] Y.-H. Chu and W. C. Chew, "A multilevel fast multipole algorithm for electrically small composite structures," Microw. Opt. Technol. Lett., vol. 43, no. 3, pp. 202-207, Nov. 2004.

[51] L. J. Jiang and W. C. Chew, "A mixed-form fast multipole algorithm," IEEE Trans. Antennas Propag., vol. 53, no. 12, pp. 4145-4156, Dec. 2005.

[52] Ö. Ergül and L. Gürel, "Efficient solutions of metamaterial problems using a low-frequency multilevel fast multipole algorithm," Progr. Electromagn. Res., vol. 108, pp. 81-99, 2010.

[53] A. K. Dominek, M. C. Gilreath, and R. M. Wood, "Almond test body," U.S. Patent 4809 003, 1989.

[54] BiLCEM Benchmarking Tool, Jul. 24, 2011. [Online]. Available: http://www.cem.bilkent. edu.tr/benchmark

\section{ABOUT THE AUTHORS}

Özgür Ergül (Member, IEEE) received the B.Sc., M.S., and Ph.D. degrees in electrical and electronics engineering from Bilkent University, Ankara, Turkey, in 2001, 2003, and 2009, respectively.

He is currently a Lecturer in the Department of Mathematics and Statistics, University of Strathclyde, Glasgow, U.K. He is also a Lecturer of the Centre for Numerical Algorithms and Intelligent Software (NAIS). He served as a Teaching and Research Assistant in the Department of Electrical and Electronics Engineering, Bilkent University, from 2001 to 2009. He was also affiliated with the Computational Electromagnetics Group, Bilkent University, from 2000 to 2005 and with the Computational Electromagnetics Research Center (BiLCEM) from 2005 to 2009. He is the coauthor of 150 journal and conference papers. His research interests include fast and accurate algorithms for the solution of electromagnetics problems involving large and complicated structures, integral equations, parallel programming, iterative methods, and high-performance computing.

Dr. Ergül is a recipient of the 2007 IEEE Antennas and Propagation Society Graduate Fellowship, the 2007 Leopold B. Felsen Award for Excellence in Electrodynamics, the 2010 Serhat Özyar Young Scientist of the Year Award, and the 2011 International Union of Radio Science (URSI) Young Scientists Award.

Levent Gürel (Fellow, IEEE) received the B.Sc. degree from the Middle East Technical University (METU), Ankara, Turkey, in 1986 and the M.S. and Ph.D. degrees in electrical and computer engineering from the University of Illinois at UrbanaChampaign (UIUC), Urbana, in 1988 and 1991, respectively.

$\mathrm{He}$ is the Director of the Computational Electromagnetics Research Center (BiLCEM), Bilkent University, Ankara, Turkey. He joined the Thomas J.

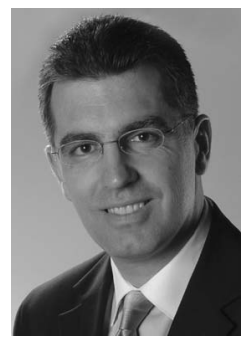
Watson Research Center of the International Business Machines Corporation, Yorktown Heights, NY, in 1991, where he worked as a Research Staff Member on the electromagnetic compatibility (EMC) problems related to electronic packaging, on the use of microwave processes in the manufacturing and testing of electronic circuits, and on the development of fast solvers for interconnect modeling. Since 1994, he has been a faculty member in the Department of Electrical and Electronics Engineering, Bilkent University, where he is currently a Professor. He was a Visiting Associate Professor at the Center for Computational Electromagnetics (CCEM) of the UIUC for one semester in 1997. He returned to the UIUC as a Visiting Professor in 2003-2005, and as an Adjunct Professor after 2005. He founded the Computational Electromagnetics Research Center (BiLCEM) at Bilkent University in 2005. His research interests include the development of fast algorithms for computational electromagnetics (CEM) and the application thereof to scattering and radiation problems involving large and complicated structures, antennas and radars, frequency-selective surfaces, highspeed electronic circuits, optical and imaging systems, nanostructures, and metamaterials. He is also interested in the theoretical and computational aspects of electromagnetic compatibility and interference analyses. Bioelectromagnetics, remote sensing, ground penetrating radars, and other subsurface scattering applications are also among his research interests. Since 2006, his research group has been breaking several world records by solving extremely large integral-equation problems.

Prof. Gürel is a Fellow of the Applied Computational Electromagnetics Society (ACES) and the Electromagnetics Academy (EMA). Among his most notable recognitions are two prestigious awards: the 2002 Turkish Academy of Sciences (TUBA) Award and the 2003 Scientific and Technical Research Council of Turkey (TUBITAK) Award. He is named an IEEE Distinguished Lecturer for 2011-2013. He is currently serving as an Associate Editor for Radio Science, the IEEE ANTENNAS AND WIRELESS Propagation LetTers, Journal of Electromagnetic Waves and Applications, and Progress in Electromagnetics Research. He is a member of the USNC of the International Union of Radio Science (URSI) and the Chairman of Commission E (Electromagnetic Noise and Interference) of URSI Turkey National Committee. He served as a member of the General Assembly of the European Microwave Association (EuMA) during 2006-2008. He is a member of the ACES Board of Directors and served as the Guest Editor for a special issue of the ACES Journal. He is invited to address the 2011 ACES Conference as a Plenary Speaker. He served as the Chairman of the AP/MTT/ED/EMC Chapter of the IEEE Turkey Section in 2000-2003. He founded the IEEE EMC Chapter in Turkey in 2000. He served as the CoChairman of the 2003 IEEE International Symposium on Electromagnetic Compatibility. He is the organizer and General Chairman of the 2007, 2009, and 2011 Computational Electromagnetics International Workshops held in Izmir, Turkey. 